\title{
Coordinating Concepts and Discourse in Model-Oriented Research Reports
}

\author{
Robert B. Allen \\ iSchool, Drexel University, Philadelphia, PA 19107, USA \\ rba@boballen.info
}

\begin{abstract}
Model-oriented research reports have been proposed as a highly structured approach that weaves together models for research methods and analyses, conceptual process models for the phenomena under investigation, and discourse structures for presenting the models.
\end{abstract}

Keywords: Argumentation, Citations, Conceptual Models, Discourse, Linked Models, Research Design, Research Procedure, Scholarly Communication

\section{Introduction}

Allen [1, 2] has proposed the development of "model-oriented scientific research reports”. Some parts of research reports are already structured but other parts have traditionally been presented just as text-based narrative. Much work on scientific communication has focused on specifying the discourse elements (which show the author's purpose). With model-oriented scientific research reports, the entire report would be structured. The model-oriented approach includes the development of conceptual process models to describe the phenomena under investigation. Discourse models and conceptual models are both essential for scientific research reports and any attempt to fully capture the content of the reports must have representations of both. There would be many advantages to model-oriented research reports. For instance, constraints are implicit in all research reports; they would be made explicit in the model-oriented approach. Moreover, recent work in digital preservation has emphasized the need to capture the details associated with scientific data sets. Some approaches explore associating the report with data as separate files. A modeloriented full research report which included the data would provide a clear basis for understanding that data. In this paper, we consider examples and issues for presenting tests of conceptual process models in model-oriented research reports.

\section{Issues for Coordinating Research Report Component Models}

We randomly selected a paper from PLoS Biology by Zhai et al. [7] as a test case to convert to a model-oriented research report. This paper presents a study exploring the molecular basis of protective factors associated with Wallerian Degeneration -- a disintegration of axons after they have been injured. The study compares two conceptual models for how the protective factors function. The two models are tested as indicated by the pseudo code below. NAD and Degeneration refer to the level of two factors in the models. The NAD level is manipulated as the independent variable and the level of Degeneration is the dependent variable. The use of conditionals for selection between model instances is related to meta-modeling, with the goal in this case to coordinate and contrast models rather than to identify a general model.

if $((\mathrm{NAD}==\mathrm{LOW}) \& \&($ Degeneration $==\mathrm{LOW}))$ then $\{$ prefer Model 1 ;

else if $((\mathrm{NAD}==\mathrm{LOW}) \& \&($ Degeneration $==$ NORMAL $)$ ) then $\{$ prefer Model2;

To capture the reasoning behind the models being tested, discourse structures need to be linked to conceptual process models. The Introduction of a research report is 
structured as defining the problem to be addressed with CARS (Creating a Research Space) [5]. In the Zhai et al. article [7], the introduction starts with an applied problem and then presents possible mechanisms of that problem to be tested. We developed a notation for describing the alternative models being tested, with a visual presentation that is highly constrained.

After the Introduction, the procedures for testing the candidate conceptual process models are described in the Methods section. The description of the testing procedures involves the coordination of several report component models. The Research Design is distinguished from the Research Procedure as a distinct component model. One of the test procedures described in the Zhai et al. paper is denoted as $\mathrm{f}_{\text {AdoptAModelSystem }}(\mathrm{)}$ - testing Drosophila as a model system for the mechanisms controlling Wallerian Degeneration in mammals. Development, validation, and application of that model system requires substantial testing, and several pages of the research report are devoted to describing the Research Procedure to do so. Showing that those pages present the Research Procedure is helpful for readers to understand the overall structure of the paper.

Citations are another area in which discourse models mix with conceptual process models. There is often ambiguity in exactly what claim is being substantiated with a citation. Consider the following example from [7]:

In an attempt to dissect the mechanisms underlying the neurodegeneration of mutant photoreceptors, we first tested whether the degeneration is light dependent, because a common cause for retinal degeneration is light stimulation [41, 42].

A reader who followed the citations would benefit from a quick, perhaps graphical, overview of the claim, the conceptual model about which the claim is being made, and how the evidence was collected to support that claim and model. For a reader who is familiar with the topic, this can sometimes be accomplished simply by skimming the cited source but it would often be more effective, especially for nonexperts, to have a highly structured overview. Indeed, interactive research reports might be developed that are personalized to the background of the readers. Moreover, the conceptual models provide a tight interweaving of the content that is not the case for most adaptive hypertexts systems.

Although deduction in science (cf., [4]) can be seen as a type of argumentation (e.g., [6]), some feel that argumentation is antithetical to deduction. Moreover, graphical argumentation systems (e.g., [3]) have not included causal models. Generally, systematic argumentation, as well as other types of discourse modeling, needs to be coupled with conceptual process models.

\section{References}

1. Allen, R.B.: Highly Structured Scientific Publications. ACM/IEEE JCDL, 472. (2007)

2. Allen, R.B.: Model-Oriented Scientific Research Reports. D-Lib, May-June, (2011)

3. Conklin, J., Begeman, M.L.: gIBIS: A Hypertext Tool for Exploratory Policy Discussion. ACM Transactions on Office Information Systems, 4, 303--331 (1988)

4. Popper, K.R.: The Logic of Scientific Discovery, Harper \& Row, New York (1968)

5. Swales, J.M.: Genre Analysis: English in Academic and Research Settings. Cambridge University Press, Cambridge UK. (1990)

6. Toulmin, S., Uses of Argument, Cambridge University Press, Cambridge UK (1958)

7. Zhai, G., Cao, Y., Hiesinger, P.R., Mehta, S.Q., Schulze, K.L., Verstreken, P., Zhou, Y., Bellen, H.J.: Drosophila NMNAT Maintains Neural Integrity Independent of its NAD Synthesis Activity. PloS Biology, 4(12) (2006) 